\title{
Who's Afraid of the Big Bad Whorf? Crosslinguistic Differences in Temporal Language and Thought
}

\author{
Daniel Casasanto
}

Stanford University

The idea that language shapes the way we think, often associated with Benjamin Whorf, has long been decried as not only wrong but also fundamentally wrong-headed. Yet, experimental evidence has reopened debate about the extent to which language influences nonlinguistic cognition, particularly in the domain of time. In this article, I will first analyze an influential argument against the Whorfian hypothesis and show that its anti-Whorfian conclusion is in part an artifact of conflating two distinct questions: Do we think in language? and Does language shape thought? Next, I will discuss crosslinguistic differences in spatial metaphors for time and describe experiments that demonstrate corresponding differences in nonlinguistic mental representations. Finally, I will sketch a simple learning mechanism by which some linguistic relativity effects appear to arise. Although people may not think in language, speakers of different languages develop distinctive conceptual repertoires as a consequence of ordinary and presumably universal neural and cognitive processes.

Are our own concepts of 'time,' 'space,' and 'matter' given in substantially the same form by experience to all men, or are they in part conditioned by the structure of particular languages? (Whorf, 1939/2000, p. 138)

Does language shape thought? Do crosslinguistic differences in the lexicon or grammar have nonlinguistic consequences, such that people who talk differently end up thinking differently in corresponding ways? If so, how does this happen: What features of language affect which cognitive processes and What are the mechanisms? Since Benjamin Whorf directed scholars' attention to this

This research was supported in part by an NSF Graduate Research Fellowship and NSF Dissertation Fellowship, by NRSA post-doctoral fellowship \#F32MH072502, and by a grant from the Spanish Ministry of Education and Science \#SEJ2006-04732/PSIC, DGI.

Correspondence concerning this article should be addressed to Daniel Casasanto, Stanford University, Jordan Hall, Bldg. 420, Stanford, CA 94305. Internet: casasanto@alum.mit.edu 
set of questions over half a century ago, few topics have incited so much passionate disagreement among linguists, anthropologists, and psychologists alike. In this article, I will not review the sanguinary history of the Whorfian debate (see Gentner \& Goldin-Meadow, 2003; Gumperz \& Levinson, 1996). Rather, I will start by disentangling two questions about the relationship between language and thought, the conflation of which may contribute to the fervor that the Whorfian hypothesis inspires in its opponents. ${ }^{1}$ Next, I will review experimental evidence that crosslinguistic differences in the lexicalization of time correlate with profound differences in the way speakers of different languages mentally represent duration. Finally, I will describe a mechanism by which language causes these differences to arise, which suggests a reframing of Whorf's question about relationships among language, concepts, and experience.

The long-standing majority view on the Whorfian hypothesis is summarized in Steven Pinker's 1994 bestseller The Language Instinct. Pinker first posed a question inspired by George Orwell's (1949) dystopian fantasy, the novel 1984, in which the government seeks to render subversive thoughts unimaginable by making them nameless in the prescribed language, Newspeak:

Is thought dependent on language? Do people literally think in English, Cherokee, Kivunjo, or by [the year] 2050, Newspeak? Or are thoughts couched in some silent medium of the brain — a language of thought or "mentalese"- and merely clothed in words whenever we need to communicate them to a listener? (p. 56)

In response, Pinker wrote:

[T]he famous Sapir-Whorf hypothesis of linguistic determinism, stating that people's thoughts are determined by the categories made available by their language, and its weaker version, linguistic relativity, that differences among languages cause differences in the thoughts of their speakers [...] is wrong, all wrong. (p. 57)

Pinker denounced not only radical linguistic determinism but also linguistic relativity, which is my concern here, and Whorf's concern in the 1939 passage quoted above. To justify his anti-Whorfian position, Pinker asserted:

The idea that thought is the same as language is an example of what can be called a conventional absurdity[.] (p. 57)

Here, Pinker illustrates a confusion that is rampant in the literature on relationships between language and thought. The idea that "thought is the same as language" (as Orwell suggested) should not be conflated with the idea that 
"differences among languages cause differences in the thoughts of their speakers" (as Whorf suggested). Orwell and Whorf raised two distinct questions: Do we think in language? and Does language shape thought? It is possible (and, I will argue, true) that language can shape the way people think even if they do not think in language.

Confusion of the Whorfian question with what I will call the "Orwellian" question pervades Pinker's discussion:

The idea that language shapes thinking seemed plausible when scientists were in the dark about how thinking works, or even how to study it. Now that scientists know how to think about thinking, there is less of a temptation to equate [thinking] with language. (pp. 58-59, italics added)

Not only does Pinker zigzag between Orwell's idea and Whorf's, he also implies a relationship between them that is misleading and, in fact, logically fallacious. The reader is led to believe that the absurdity of the Orwellian proposal should be taken as one of the strongest arguments against the Whorfian hypothesis. To unpack this fallacy, let us turn the Orwellian and Whorfian questions into two statements, $\mathrm{O}$ and $\mathrm{W}[(1 \mathrm{a})$ and (b)]:

(1a) O: We think in language.

(1b) W: Language shapes thought.

There is a clear relationship between these statements. If people think in language, then it must be the case that language shapes thought. In other words, if Orwell was right, then Whorf must necessarily be right, too. This can be expressed in the proposition (2): if $\mathrm{O}$, then $\mathrm{W}$.

\section{(2) $\mathrm{O} \rightarrow \mathrm{W}$}

If we agree to the truth of this proposition, then we must also agree to the truth of its contrapositive (3): If language does not shape thought, then we do not think in language. In other words, proving that Whorf was wrong would entail that Orwell was wrong, as well.

$$
\sim \mathrm{W} \rightarrow \sim \mathrm{O}
$$

However, this is not what Pinker argued. Rather, he suggested that people do not think in language; therefore, language does not shape thought. In other words, because Orwell was wrong, we should believe that Whorf must be wrong. However, this argument assumes that the inverse (4) of our proposition is true, a logical fallacy known as "denying the antecedent." 


$$
\sim \mathrm{O} \rightarrow \sim \mathrm{W}
$$

It may sound reasonable at first to say that "if people think in language, then language shapes thought, but because people do not think in language, then language does not shape thought"-provided you do not think about it too carefully. In fact, this is equivalent to saying, "if John has a daughter who plays the violin, then he must be a human, but because John does not have a daughter who plays the violin, then he is not human."

Although the Orwellian question and the Whorfian question are importantly related, they are not related in the way that Pinker suggested (and he is not alone in making this logical error). Evidence in favor of Orwell's idea would also support Whorf's, but evidence against the idea that people think in language does not count against the possibility that language shapes thought. Clarifying this relationship is important: If people believe that linguistic relativity entails (or implies) the Orwellian notion that people think in language, then no wonder they are afraid of the big bad Whorf!

\section{Orwell and Whorf: Divide and Conquer?}

Having distinguished the Orwellian and Whorfian questions, it is possible to evaluate them separately. Pinker's 1994 discussion, which he later dubbed his "obituary" for the Whorfian hypothesis (Pinker, 2007, p. 124), reviews standard arguments against both the idea that we think in language and the idea that language shapes thought. Although arguments against the one idea are often misrepresented as arguments against the other, many of these objections are compelling when properly construed. Pinker takes a shotgun approach to discrediting the Orwellian equation of language with thought, starting with appeals to intuition: We have all had the feeling that we know what we want to say but we do not know how to say it, therefore there must be some difference between what we say and what we want to say. Other arguments stress the inadequacy of language as a medium for thought, pointing to problems such as ambiguity, deixis, and coreference, which seem to require extralinguistic resources to resolve. For example, when we read the ambiguous headline "Hershey Bars Protest" (Pinker, 1994, p. 119), the information we need to decide whether this

story discusses an oppressive chocolate manufacturer or some rebellious candy bars does not appear to reside in language, per se. Still other arguments call upon results from psychological experiments, such as Roger Shepard and Lynn Cooper's (1982) studies suggesting that people rotate objects in their mind's eye using imagistic, picturelike representations. Most compellingly, Pinker pointed 
out that much of what can reasonably be construed as "thinking" happens in the minds of babies, deaf isolates, and aphasics, who lack the full use of language, and even in the minds of animals that lack language altogether. The fact that animals can learn, remember, navigate, communicate, reason about causes, infer goals, build shelters, use tools, cooperate, deceive one another, and construct social hierarchies suggests that quite a lot of thinking can happen without human language.

These anti-Orwellian arguments do not necessarily support Pinker's conclusion that all thinking occurs in a Fodorian "mentalese" (Fodor, 1975). They do not rule out the possibility that certain kinds of thinking are mediated by language (see Carruthers, 1996; Spelke \& Tsivkin, 2001). They do suggest, however, that even if some thinking takes place in the medium of natural language, not all thinking does, and that the Orwellian proposal is problematic, in principle.

Standard arguments against the Whorfian hypothesis, including Pinker's, are of a different sort. Rather than attacking linguistic relativity in principle, they criticize the data and methods that have been used to support Whorfian claims. Do the Eskimos really think about snow more precisely than speakers of a Standard Average European (SAE) language because they can categorize it more precisely in language (Whorf, 1940/2000)? Are Hopi speakers unable to conceptualize time as we do because of their impoverished temporal vocabulary (Whorf, 1939/2000)? There is no good evidence that this is the case. Two things were wrong with some of Whorf's most notorious claims. To start, many of Whorf's linguistic observations did not stand up to scrutiny by later scholars. Arguably, speakers of Eskimo languages do not use any more words for snow than your average snowboarding enthusiast (Pullum, 1991), and the Hopi speakers' way of talking about time may not be as different from the SAE speakers' as Whorf made it seem (Malotki, 1983). If there are not actually any relevant differences between languages, then there is no reason to posit differences in the thoughts of their speakers. Furthermore, the project of inferring cognitive differences solely from linguistic differences is hopelessly circular. Patterns in language can serve as a source of hypotheses about cognitive differences between members of different language communities, but some sort of extralinguistic data are needed to test these hypotheses: Otherwise, the only evidence that people who talk differently also think differently is that they talk differently!

Experimental work since Whorf's time has suffered several additional problems. Pinker noted that some apparent behavioral differences between language groups have turned out to be artifacts of clumsy translation. Famously, Alfred 
Bloom (1981) alleged that Chinese speakers are less capable of reasoning counterfactually than English speakers because Chinese lacks subjunctives, which serve as counterfactual markers in English (e.g., If it were to rain, then I would take an umbrella). In his original experiment, Bloom created English and Chinese versions of a story that required counterfactual reasoning, and he found that Chinese speakers in China failed abysmally to understand the story's counterfactual structure, whereas English speakers in the United States had no trouble at all. Terry Au, a native Chinese-speaking psychologist, examined the stories that Bloom had used and found the Chinese version to be "not very idiomatic" (Au, 1983, p. 161). When Au replicated Bloom's experiment using new stimuli, the crosslinguistic differences in counterfactual reasoning disappeared $(\mathrm{Au})$.

Yet, awkward translations may be the least of the problems exemplified by Bloom's study (and found in many subsequent studies, as well). No matter how carefully stimulus materials are translated, any experimental design that relies on comparing performance across translations confounds differences between items with differences between conditions (and if monolinguals are used, also confounds item differences with group differences), making the results hard to interpret. Furthermore, this study predicted a single difference between language groups: One group should perform better than the other. Even if the predicted difference had been reliable (e.g., if English speakers had shown better counterfactual reasoning than Chinese speakers across studies), it would be hard to know why: the group that is better at counterfactual reasoning could also be better at many other kinds of reasoning that do not correspond to any crosslinguistic differences. Unless predictions are cast in terms of statistical interactions, researchers risk interpreting spurious relationships between patterns in language and patterns in performance on experimental tasks. Finally, the Whorfian hypothesis posits a causal relationship between language and thought. Bloom sought to test the hypothesis that language causes differences in counterfactual reasoning using an experimental design that was only capable of demonstrating correlation - a problem that is not intractable, but which still plagues would-be Whorfian research a quarter of a century later (see Casasanto, 2005a; Gordon, 2004).

The Orwellian idea that people think (mostly or entirely) in the medium of natural language, and therefore that language can be equated with thought, is unsupported empirically and is also problematic in principle, given what is known about language and about thought. ${ }^{2}$ By contrast, the Whorfian idea that linguistic differences can cause speakers of different languages to think differently faces no such in-principle challenges. When standard arguments 
are properly interpreted, they do not sound a death knell for the Whorfian hypothesis, but rather a call for more rigorous research.

\section{Time in Language and Thought}

Since Pinker's (1994) "obituary," Whorfian research has experienced a renaissance. Experimental evidence has reopened debate about the extent to which language influences nonlinguistic cognition in domains such as space (Levinson, 1996; Li \& Gleitman, 2002; Majid, Bowerman, Kita, Haun, \& Levinson, 2004), color (Gilbert, Regier, Kay, \& Ivry, 2006; Kay \& Kempton, 1984; Robertson, Davies, \& Davidoff, 2000; Witthoft, et al., 2003), number (Casasanto, 2005a; Gordon, 2004; Gelman \& Gallistel, 2004; Miller, Major, Shu, \& Zhang, 2000; Pica, Lemer, Izard, \& Dehaene, 2004; Spelke \& Tsivkin, 2001), and time (Boroditsky, 2001; Casasanto et al., 2004; Chen, 2007; January \& Kako, 2007; Núñez \& Sweetser, 2006). One obstacle to resolving this controversy has been devising truly nonlinguistic tests to evaluate how speakers of different languages perceive or remember their experiences, particularly in the more abstract conceptual domains such as time.

Across languages, people use the same words to talk about time that they use to talk about space (Alverson, 1994; Clark, 1973; Gruber, 1965; Haspelmath, 1997; Jackendoff, 1983; Lakoff \& Johnson, 1980; Traugott, 1978). For example, English speakers might talk about a long vacation or a long line and moving a meeting forward or moving a truck forward. Evidence from behavioral experiments suggests that people not only talk about time using spatial language, they also think about time using mental representations of space (Boroditsky, 2000, 2001; Boroditsky \& Ramscar, 2002; Casasanto, 2005b, in press; Casasanto \& Boroditsky, 2003, 2008; Casasanto et al., 2004; Cohen, 1967; Gentner, 2001; Núñez \& Sweetser, 2006; Piaget, 1927/1969; Torralbo, Santiago, \& Lupiáñez, 2006; Tversky, Kugelmass, \& Winter, 1991). Although using spatial metaphors for time may be universal (Alverson, 1994; cf. Silva Sinha, Sinha, Zinken, \& Sampaio, 2008), the particular mappings from space to time vary across languages. For instance, depending on the language, speakers might talk about the future as if it lies ahead of us (in English), behind us (in Aymara), or below us (in Mandarin Chinese). Behavioral studies suggest that speakers of languages that use different spatiotemporal metaphors may indeed think about time differently (Boroditsky, 2001; Núñez \& Sweetser, 2006).

There is, however, an important limitation shared by these crosslinguistic studies comparing English speakers' mental representations of time with those 
of Aymara and Mandarin speakers: Subjects were tested on tasks that required them to produce or understand language. Núñez and Sweetser's participants were producing co-speech gestures, and Boroditsky's were judging sentences containing spatial or temporal language. Perhaps these studies showed relationships between spatial and temporal thinking that were consistent with linguistic metaphors only because participants were required to process space or time in language (i.e., because they were "thinking for speaking"; Slobin, 1996). Would the same relationships between mental representations of space and time be found if participants were tested on nonlinguistic tasks?

To address this question, my collaborators and I developed simple psychophysical tasks to investigate whether metaphors in language influence even our basic, perceptuomotor representations of time. Do speakers of languages that use different spatiotemporal metaphors think about time differently-even when they are not using language? First, we analyzed previously unexplored crosslinguistic differences in metaphors for duration in English and Greek. Next, we tested whether these linguistic differences correlate with differences in English and Greek speakers' performance on low-level, nonlinguistic duration estimation tasks. Finally, we conducted a training study to evaluate a causal role for language in shaping time representations (Casasanto, 2005b; Casasanto et al., 2004).

\section{Duration in One or Three Dimensions}

Literature on how time can be expressed verbally in terms of space (and, by hypothesis, conceptualized in terms of space) has focused principally on linear spatial metaphors. However, is time necessarily verbalized (and conceptualized) in terms of unidimensional space? Some theorists have suggested so (Clark, 1973; Gentner, 2001), and although this may be true regarding temporal succession, linguistic metaphors suggest an alternative spatialization for duration. English speakers not only describe time as a line, but they also talk about having oceans of time, saving time in a bottle, and liken the days of their lives to sands through the hourglass. Quantities of time are described as amounts of a substance that occupies three-dimensional space (i.e., volume).

Languages differ in the extent to which they describe duration in terms of distance as opposed to amount of substance. In English, it is natural to talk about a long time or a long meeting, borrowing the structure and vocabulary of a linear spatial expression like a long rope. In Greek, the words makris and kontos are the literal equivalents of the English spatial terms long and short. They can be used in spatial contexts much the way long and short are used in English (e.g., ena makry skoini means "a long rope"). In temporal 
contexts, however, makris and kontos are dispreferred in many instances where long and short would be used naturally in English. It would be unnatural to translate "a long meeting" literally as "mia makria synantisi." Rather than using distance terms, Greek speakers typically indicate that an event lasted a long time using megalos, which in spatial contexts means physically large (e.g., a big building), or using poli, which in spatial contexts means much (e.g., much water).

To quantify the relative prevalence of "distance" and "amount" metaphors for duration across languages, the frequencies of the English phrases long time and much time were compared with the Greek phrases that native speakers reported most naturally expressed the same ideas, makry kroniko diastima "long time distance" and poli ora "much time." Each expression was entered as a search term in a very large multilingual text corpus (www.google.com). Search results showed that the distance metaphor was dramatically more frequent than the amount metaphor in English, whereas the opposite pattern was found in Greek. Results were corroborated in a questionnaire study showing that English speakers tend to use distance metaphors to describe the duration of events (e.g., long meeting, long party), whereas Greek speakers tend to use amount metaphors to describe the durations of the same events (e.g., synantisi pou diekese poli "meeting that lasts much," parti pou kratise poli "party that lasts much"). Although both languages use some distance and some amount metaphors for duration, the relative strengths of these metaphors varies across languages. These preliminary studies by no means captured all of the complexities of how duration is metaphorized in terms of space within or between languages, but findings corroborated native English and Greek speakers' intuitions and provided quantitative linguistic measures on which to base predictions about behavior in nonlinguistic tasks.

Do these differences in metaphor frequency between languages lead English and Greek speakers to think about time differently, even when they are not using language? To find out, we asked English and Greek speakers to estimate the duration of brief events that contained either distracting information about linear distance (distance interference) or distracting information about amount (amount interference). In the distance interference condition, participants viewed a series of lines "growing" across the screen, for various distances and durations. After each line disappeared, they were asked to reproduce its duration by clicking the mouse twice (in the same spot) to indicate the time that elapsed from the instant that the line appeared to the instant that it reached its maximum length. The distance that the line grew was irrelevant to the task and was varied orthogonally to the line's duration. As such, distance served 
as distractor: a piece of information that was unrelated to the task but could potentially interfere with task performance. In the amount interference condition, subjects viewed a schematic drawing of a container filling gradually with liquid and were asked to reproduce the duration of the "filling" event. Analogously to the distance interference condition, the amount of fill varied orthogonally with the duration of the event and, as such, served as a distractor for the subjects' task of estimating duration.

In previous studies using a similar distance interference task with English speakers (Casasanto \& Boroditsky, 2003, 2008), English-speaking participants were unable to ignore the distance that a line grew when estimating its duration, even though distance was irrelevant to the time estimation task. The result was a pattern of cross-dimensional interference: Spatial information interfered with participants' temporal judgments in a particular way. Although, on average, all stimuli lasted the same duration, participants judged lines that traveled a shorter distance to last a shorter time and lines that traveled a longer distance to last a longer time.

Is this conflation of distance and duration universal to humans, or does it depend in part on the conflation of distance and duration in language? If patterns in language are partly responsible for the space-time confusion observed in English speakers, then irrelevant distance and amount information should interfere with English and Greek participants' duration estimates differently. English speakers should show more interference from distance than amount on their time estimates. Greek speakers should show the opposite pattern, being more distracted by amount than by distance interference. Results supported these predictions: The pattern of cross-dimensional interference observed in English and Greek speakers on this pair of nonlinguistic time estimation tasks followed the pattern of spatiotemporal metaphors found in English and Greek. English speakers were strongly affected by the distance that a line traveled but only weakly affected by the fullness of a container, whereas Greek speakers showed the opposite pattern of cross-dimensional interference. The structure of people's low-level, nonlinguistic time representations is not universal: These simple psychophysical tasks indicate that at a basic level, the way we mentally represent time covaries with the way we talk about it in our native languages. However, does experience using different metaphors cause speakers of different languages to think differently?

\section{How Language Shapes Time}

Previous suggestions for how language could influence representations of time have failed because they have attempted to give an Orwellian answer to a 
Whorfian question, equating concepts with words. Paul Bloom and Frank Keil (2001) created the following straw-man, which makes the Whorfian proposal appear hopelessly circular:

Since the lexicons of languages differ, it would follow that speakers of different languages would come to possess different concepts. How coherent is this proposal? Consider a simple example:

Q: How is it that people can think about time?

A: Because we learn the language of time, words like 'was' and 'tomorrow.'

But this answer immediately raises another question: How do we learn these words? (pp. 361-362)

Bloom and Keil (2001) argued that the ability to learn temporal words presupposes an ability to think about time, thus begging the question of from where this ability comes. However, why should the Whorfian claim that lexical differences create conceptual differences entail that words precede concepts? Language can influence the structure and content of preexisting mental representations via simple learning mechanisms, one of which I will illustrate here.

How do Greek and English speakers come to mentally represent duration differently, relying differentially on mental representations of distance or amount? It is not plausible that using temporal metaphors in language creates the capacity to estimate brief durations, because prelinguistic infants and nonhuman animals share this capacity. Consider, instead, that some mappings from concrete to abstract domains of knowledge (such as mappings from space to time) may be initially established prelinguistically, based on interactions with the physical world (Clark, 1973). As an example, people are likely to track the kinds of correlations in experience that are important for perceiving and acting on their environment; they learn to associate time with linear space by observing that more time passes as moving objects travel farther, and likewise they learn to associate time with amounts of substances accumulating in three-dimensional space by observing that more time passes as substances accumulate more. This proposal presupposes that although mature time representations depend in part on spatial representations, time can also be mentally represented qua time, at least initially: In order for cross-dimensional associations to form, some primitive representations must already exist in each dimension. Primitive temporal notions, however, of the sort that we share with infants and nonhuman animals may be too vague or fleeting to support higher order reasoning about time. Grafting primitive temporal representations onto spatial representations may 
make time more amenable to verbal or imagistic coding and may also import the inferential structure of spatial relations into the domain of time (Pinker, 1997), facilitating the comparison of temporal intervals, transitive inference, serial ordering, and other such mental operations that humans have evolved to perform in the domain of space.

If metaphorical mappings from space to time are based on physical experiences and are established prelinguistically, what role might language play in shaping temporal representations? Because the laws of physics are the same in all language communities, prelinguistic children's conceptual mappings between time and distance and between time and amount should be the same universally. Later, as children acquire language, these mappings are adjusted: Each time we use a linguistic metaphor, we activate the corresponding conceptual mapping. Speakers of "distance languages" like English then activate the time-distance mapping frequently, eventually strengthening it at the expense of the time-amount mapping (and vice versa for speakers of "amount languages" like Greek). At a neural level, long-term strengthening of the more frequent association at the expense of the less frequent association could be mediated by competitive Hebbian learning, and short-term adjustments in the strength of these mappings (due to immediate physical or linguistic experience) could be mediated by more transient neuromodulatory processes.

Did linguistic experience give rise to language-related differences in performance on the "growing line" and "filling container" experiments? Using crosslinguistic data to test for a causal influence of language on thought is problematic, as the experimenter cannot randomly assign participants to have one first language or another: Crosslinguistic studies are necessarily quasiexperimental. A pair of training tasks (i.e., true experimental interventions) was conducted to provide an in-principle demonstration that language can influence even the kinds of low-level mental representations that people construct while performing psychophysical tasks and to test the proposal that language shapes time representations, both in the laboratory and in natural settings, by adjusting the strengths of cross-domain mappings. Native English speakers were randomly assigned to perform either a "distance training" or "amount training" task. Participants completed 192 fill-in-the-blank sentences using the words longer or shorter for distance training and the words more or less for the amount training task. Half of the sentences compared the length or capacity of physical objects (e.g., An alley is longer/shorter than a clothesline; A teaspoon is more/less than an ocean) and the other half compared the duration of events (e.g., A sneeze is longer/shorter than a vacation; A sneeze is more/less than a 
vacation). By using distance terms to compare event durations, English speakers in the control condition were reinforcing the already preferred mapping between distance and time. By using amount terms, participants in the critical condition were describing event durations similarly to speakers of an amountbiased language like Greek, activating the nonlinguistic amount-time mapping that is normally dispreferred for English speakers. After this linguistic training phase, all participants performed the nonlinguistic filling container task. If using a linguistic metaphor activates the corresponding conceptual mapping, then repeatedly using amount metaphors during training should transiently strengthen participants' nonlinguistic amount-time mapping. Consistent with this prediction, following about $30 \mathrm{~min}$ of training with amount metaphors, native English speakers' performance on the filling container task was statistically indistinguishable from the performance of the native Greek speakers. By encouraging the habitual activation of either distance-time or amount-time conceptual mappings, our experience with natural language may influence our everyday thinking about time in much the same way as this laboratory training task.

In summary, people who talk differently about time also think about it differently, in ways that correspond to the preferred metaphors in their native languages. Language not only reflects the structure of our temporal representations, but it can also shape those representations. Beyond influencing how people think when they are required to speak or understand language, language can also shape our basic, nonlinguistic perceptuomotor representations of time. It may be universal that people conceptualize time according to spatial metaphors, but because these metaphors vary across languages, members of different language communities develop distinctive conceptual repertoires.

However, there is no need to be afraid of this Whorfian effect. The fact that language influences thought does not mean that people think in language, nor does it imply that language interfaces with nonlinguistic mental representations via privileged channels or special mechanisms: In this case, associative learning will do. Whorf asked whether our concepts are given in the same form by experience to everyone or whether they are conditioned by language. The results summarized here suggest that conceptual mappings from space to time may be given in essentially the same form via correlations in physical experience to everyone and then also conditioned by the languages we speak. If language plays a privileged role in shaping thought, it is perhaps only by virtue of being a ubiquitous and highly systematic form of experience, which, unlike basic perceptuomotor experiences, varies from culture to culture. 


\section{Why Worry About Whorf?}

Why should we continue to do Whorfian research? One possible reason is that cataloging crosslinguistic cognitive differences could be a step toward charting the boundaries of human biological and cultural diversity. If this is the goal, then the Whorfian effects most worth finding should be extreme instances in which differences between languages produce radically different experiences of reality in their speakers. Alternatively, crosslinguistic cognitive differences could be tools for investigating how thinking works and, in particular, for investigating the role of experience in the acquisition and representation of knowledge: If people who talk differently form correspondingly different mental representations as a consequence, then mental representations must depend, in part, on these aspects of linguistic experience. If discovering the origin and structure of our mental representations is the goal, then crosslinguistic cognitive differences can be informative even if they are subtle and even if their effects are largely unconscious. Whether or not they correspond to radical differences in speakers' conscious experiences of the world, Whorfian effects can have profound implications for the study of mental representation.

\section{Notes}

1 I will use the term "Whorfian hypothesis" to mean the linguistic relativity hypothesis, that differences among languages cause differences in the thoughts of their speakers, as suggested by the question of Whorf's quoted at the beginning of the article. Arguments supporting linguistic relativity should not be interpreted as supporting linguistic determinism, the idea that language rigidly determines the thoughts that speakers are capable of entertaining, which has also been associated with Whorf.

2 The "Orwellian" claim that people think in language therefore language can be equated with thought, is sometimes labeled strong linguistic determinism and attributed to Whorf. I assert that nothing Whorf wrote supports this attribution, although defending this assertion would require a full exegesis of his more radical statements, which I will not undertake here. More importantly, linguistic determinism, the idea that "people's thoughts are determined by the categories made available by their language" (Pinker, 1994, p. 57), does not entail that "the medium of thought consists of the actual words and sentences the person speaks" (Pinker, 2007, p. 133). Language could still dictate "the categories and types that we isolate from the world of phenomena" (Whorf, 1940/2000, p. 213) even if thinking is not carried out in the medium of natural language. There is no empirical evidence for strong linguistic determinism, but none of the arguments that I have reviewed against the Orwellian idea rules out linguistic determinism, in principle. 


\section{References}

Alverson, H. (1994). Semantics and experience: Universal metaphors of time in English, Mandarin, Hindi, and Sesotho. Baltimore: Johns Hopkins University Press.

$\mathrm{Au}, \mathrm{T}$. (1983). Chinese and English counterfactuals: The Sapir-Whorf hypothesis revisited. Cognition, 15, 155-187.

Bloom, A. (1981). The linguistic shaping of thought: A study in the impact of language on thinking in China and the West. Hillsdale, NJ: Lawrence Erlbaum Associates.

Bloom, P., \& Keil, F. C. (2001). Thinking through language. Mind and Language, 16(4), 351-367.

Boroditsky, L. (2000). Metaphoric structuring: Understanding time through spatial metaphors. Cognition, 75(1), 1-28.

Boroditsky, L. (2001). Does language shape thought? Mandarin and English speakers' conceptions of time. Cognitive Psychology, 43(1), 1-22.

Boroditsky, L., \& Ramscar, M. (2002). The roles of body and mind in abstract thought. Psychological Science, 13(2), 185-189.

Carruthers, P. (1996). Language, thought and consciousness. Cambridge: Cambridge University Press.

Casasanto, D. (2005a). Crying “Whorf!” Science, 307, 1721-1722.

Casasanto, D. (2005b). Perceptual foundations of abstract thought. Unpublished doctoral dissertation, MIT, Cambridge, MA.

Casasanto, D. (in press). Space for thinking. In V. Evans \& P. Chilton (Eds.), Language, cognition and space: State of the art and new directions. London: Equinox Publishing.

Casasanto, D., \& Boroditsky, L. (2003). Do we think about time in terms of space? In R. Alterman \& D. Kirsh (Eds.), Proceedings of 25th Annual Conference of the Cognitive Science Society (pp. 216-221). Hillsdale, NJ: Lawrence Erlbaum Associates.

Casasanto, D., \& Boroditsky, L. (2008). Time in the mind: Using space to think about time. Cognition, 106(2), 579-593.

Casasanto, D., Boroditsky, L., Phillips, W., Greene, J., Goswami, S., Bocanegra-Thiel, et al. (2004). How deep are effects of language on thought? Time estimation in speakers of English, Indonesian, Greek, and Spanish. In K. Forbus, D. Gentner, \& T. Regier (Eds.), Proceedings of the 26 h Annual Conference Cognitive Science Society (pp. 575-580). Hillsdale, NJ: Lawrence Erlbaum Associates.

Chen, J. (2007). Do Chinese and English speakers think about time differently? Failure of replicating Boroditsky (2001). Cognition, 104(2), 427-436.

Clark, H. H. (1973). Space, time, semantics and the child. In T. E. Moore (Ed.), Cognitive development and the acquisition of language (pp. 27-63). New York: Academic Press.

Cohen, J. (1967). Psychological time in health and disease. Springfield, IL: Charles C Thomas. 
Fodor, J. (1975). The language of thought. Cambridge, MA: Harvard University Press. Gelman, R., \& Gallistel, C. R. (2004). Language and the origin of numerical concepts. Science, 306, 441-443.

Gentner, D. (2001). Spatial metaphors in temporal reasoning. In M. Gattis (Ed.), Spatial schemas and abstract thought (pp. 203-222). Cambridge, MA: MIT Press.

Gentner, D., \& Goldin-Meadow, S. (2003) Language in mind: Advances in the study of language and thought. Cambridge, MA: MIT Press

Gilbert, A., Regier, T., Kay, P., \& Ivry, R. B. (2006). Whorf hypothesis is supported in the right visual field but not the left. Proceedings of the National Academy of Sciences USA 103(2), 489-494.

Gordon, P. (2004). Numerical cognition without words: Evidence from Amazonia. Science, 306, 496.

Gruber, J. (1965). Studies in lexical relations. Unpublished doctoral dissertation, MIT, Cambridge, MA.

Gumperz, J. J., \& Levinson, S. C. (1996). Rethinking linguistic relativity. Cambridge: Cambridge University Press.

Haspelmath, M. (1997). From space to time: Temporal adverbials in the world's languages. Munich: Lincom Europa.

Jackendoff, R. (1983). Semantics and cognition. Cambridge, MA: MIT Press.

January, D., \& Kako, E. (2007). Re-evaluating evidence for linguistic relativity: Reply to Boroditsky (2001). Cognition, 104(2), 417-426.

Kay, P., \& Kempton, W. (1984). What is the Sapir-Whorf hypothesis? American Anthropologist, 86(1), 65-79.

Lakoff, G., \& Johnson, M. (1980). Metaphors we live by. Chicago: University of Chicago Press.

Levinson, S. C. (1996). Frames of reference and Molyneux's question: Cross-linguistic evidence. In P. Bloom, M. Peterson, L. Nadel, \& M. Garrett (Eds.), Language and space: Language, speech, and communication (pp. 385-436). Cambridge, MA: MIT Press.

Li, P., \& Gleitman, L. (2002). Turning the tables: Language and spatial reasoning. Cognition, 83(3), 265-294.

Majid, A., Bowerman, M., Kita, S., Haun, D. B. M., \& Levinson, S. C. (2004). Can language restructure cognition? The case for space. Trends in Cognitive Sciences, $8(3), 108-114$.

Malotki, E. (1983). Hopi time: A linguistic analysis of temporal concepts in the Hopi language. Berlin: Mouton.

Miller, K., Major, S., Shu, H., \& Zhang, H. (2000). Ordinal knowledge: Number names and number concepts in Chinese and English. Canadian Journal of Experimental Psychology, 54(2), 129-139.

Núñez, R., \& Sweetser, E. (2006). Looking ahead to the past: Convergent evidence from Aymara language and gesture in the crosslinguistic comparison of spatial construals of time. Cognitive Science, 30(3), 401-450. 
Orwell, G. (1949). Nineteen eighty-four. A novel. London: Secker \& Warburg.

Piaget, J. (1927/1969). The child's conception of time. New York: Ballantine Books.

Pica, P., Lemer, C., Izard, V., \& Dehaene, S. (2004). Exact and approximate arithmetic in an Amazonian indigene group. Science, 306, 499-503.

Pinker, S. (1994). The language instinct: How the mind creates language. New York: Harper.

Pinker, S. (1997). How the mind works. New York: Norton.

Pinker, S. (2007). The stuff of thought: Language as a window into human nature. New York: Viking.

Pullum, G. K. (1991). The great Eskimo vocabulary hoax and other irreverent essays on the study of language. Chicago: University of Chicago Press.

Robertson, D., Davies, I., \& Davidoff, J. (2000). Color categories are not universal: Replications and new evidence from a stone-age culture. Journal of Experimental Psychology: General, 129(3), 369-398.

Shepard, R. N., \& Cooper, L. A. (1982). Mental images and their transformations. Cambridge, MA: MIT Press.

Silva Sinha, V., Sinha, C., Zinken, J., \& Sampaio, W. (2008) When time is not space. The social and linguistic construction of time intervals in an Amazonian culture. Manuscript submitted for publication.

Slobin, D. I. (1996). From "thought and language" to "thinking for speaking." In J. J. Gumperz \& S. C. Levinson (Eds.), Rethinking linguistic relativity (pp. 70-96). Cambridge: Cambridge University Press.

Spelke, E., \& Tsivkin, S. (2001). Language and number: A bilingual training study. Cognition, 78(1), 45-88.

Torralbo, A., Santiago, J., \& Lupiáñez, J. (2006). Flexible conceptual projection of time onto spatial frames of reference. Cognitive Science, 30(4), 745-757.

Traugott, E. (1978). On the expression of spatiotemporal relations in language. In J. Greenberg (Ed.), Universals of human language: Word structure (Vol. 3, pp. 369-400). Stanford, CA: Stanford University Press.

Tversky, B., Kugelmass, S., \& Winter, A. (1991). Cross-cultural and developmental trends in graphic productions. Cognitive Psychology, 23(4), 515-557.

Whorf, B. L. (2000). The relation of habitual thought and behavior to language. In J. B. Carroll (Ed.), Language, thought and reality: Selected writings of Benjamin Lee Whorf (pp. 134-159). Cambridge, MA: MIT Press. (Original work published 1939)

Whorf, B. L. (2000). Science and linguistics. In J. B. Carroll (Ed.), Language, thought and reality: Selected writings of Benjamin Lee Whorf (pp. 207-219). Cambridge, MA: MIT Press. (Original work published 1940)

Witthoft, N., Winawer, J., Wu, L., Frank, M., Wade, A., \& Boroditsky, L. (2003). Effects of language on color discriminability. In R. Alterman \& D. Kirsh (Eds.), Proceedings of the 25th Annual Conference of the Cognitive Science Society (pp. 1247-1252). Hillsdale, NJ: Lawrence Erlbaum Associates. 